\title{
Reflets
}

Revue ontaroise d'intervention sociale et communautaire

\section{Un regard sur diverses violences}

\section{Myriam Bals}

Volume 13, numéro 1, 2007

La violence dans tous ses états

URI : https://id.erudit.org/iderudit/016810ar

DOI : https://doi.org/10.7202/016810ar

Aller au sommaire du numéro

Éditeur(s)

Reflets : Revue ontaroise d'intervention sociale et communautaire

ISSN

1203-4576 (imprimé)

1712-8498 (numérique)

Découvrir la revue

Citer ce document

Bals, M. (2007). Un regard sur diverses violences. Reflets, 13(1), 10-15.

https://doi.org/10.7202/016810ar

Tous droits réservés (C) Reflets : Revue ontaroise d'intervention sociale et communautaire, 2007

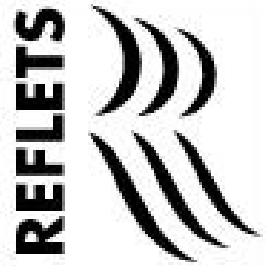

Ce document est protégé par la loi sur le droit d'auteur. L'utilisation des services d'Érudit (y compris la reproduction) est assujettie à sa politique d'utilisation que vous pouvez consulter en ligne.

https://apropos.erudit.org/fr/usagers/politique-dutilisation/ 


\section{Un regard sur diverses violences}

\section{Myriam Bals}

La violence dans tous ses états. Pourquoi un tel thème? Tout simplement parce que la violence revêt plusieurs formes et que certaines sont souvent oubliées, négligées. Les violences sont présentes aussi bien au niveau structurel que systémique et qu'individuel. Entre la violence étatique et la violence interpersonnelle, nous avons un large éventail de violences, orientées vers des groupes ou des individus. Cette violence peut être physique et laisser des traces très visibles ou, au contraire, très pernicieuse et cachée, laissant des traces psychologiques. Certaines des formes plus pernicieuses de violence ne commencent à être reconnues que depuis très récemment. De façon implicite ou explicite, la notion de genre traverse les divers articles; la violence est perpétrée contre les personnes perçues comme vulnérables, ainsi que celles apparentées au genre féminin. Il n'est pas étonnant que les personnes immigrantes ou réfugiées, les femmes, les enfants et les hommes homosexuels soient les principales victimes de ces diverses formes de violence. Quelle que soit sa forme, la violence crée des souffrances, enfermant simultanément ses victimes dans la solitude.

Ce numéro de Reflets va donc nous exposer à quelquesunes des multiples facettes de la violence, qui peut être à la fois systémique et interpersonnelle. Certains auteurs vont nous montrer la violence résultant ou faisant partie de pays en guerre, et d'autres, les conséquences sur ces ressortissants qui arrivent au Canada, espérant y commencer une nouvelle vie pour eux et leurs enfants. Cependant, même si le Canada n'est pas en guerre, ces personnes immigrantes doivent passer par beaucoup d'épreuves, ainsi que leurs enfants qui peuvent connaitre le racisme à l'école.

Cette violence ne laisse pas ses victimes sans séquelles et met à l'épreuve leur capacité de résilience. Elle peut rendre les 
victimes vulnérables et peut les empêcher de solliciter une aide extérieure. Cette incapacité de demander de l'aide peut être renforcée par la culture d'origine, mais également par sa relation aux autres, la capacité et surtout l'incapacité d'avoir confiance aux autres y compris en l'intervenante ou intervenant, le plus souvent " étranger» dans tous les sens du terme. Souvent issues de pays où la violence est organisée et systémique, ces personnes ressortissantes ont besoin d'intervenantes ou d'intervenants conscients de leurs besoins et de leurs réalités, d'intervenantes ou d'intervenants compréhensifs et patients qui acceptent que la confiance ne puisse se développer qu'avec le temps.

\section{Le dossier}

Marie Lacroix et Charlotte Sabbah procèdent à une analyse très sensible de la violence sexuelle faite contre les femmes dans les pays en guerre et vivant avec des conflits ethniques, laquelle analyse débouche sur une réflexion portant sur les défis que cela pose dans la pratique. Ce n'est que dans les années 90 que ce genre de violence a commencé à être reconnu et que ces femmes abusées ont demandé le statut de réfugiées sur cette base. Selon ces auteures, la compréhension de la violence sexuelle dans un contexte de guerre et de conflits ethniques doit comprendre les trois axes d'analyse suivants : «1) Le contexte moderne des guerres et des conflits ethniques; 2) la définition de la violence contre les femmes selon un cadre intersectionnel de relations de genre et de constructions culturelles; 3) les conséquences psychologiques, sociales et physiques de la violence sexuelle et les implications pour la pratique.» Le viol de guerre est désormais nommé et reconnu, ce qui ne permet pourtant pas toujours à ses victimes de bénéficier de services adéquats et culturellement sensibles, car ce sujet ne peut être compris et l'intervention pertinente effectuée qu'en incluant la dimension culturelle. Comment parler d'un tel traumatisme quand notre culture ne le permet pas, même si les 
"Occidentaux » reconnaissent désormais cette forme de violence contre les femmes?

Les mêmes défis de la pratique sont développés par Lamine Diallo et Ginette Lafrenière, dans leur réflexion sur l'intervention auprès des survivants de guerre, torture et violence organisée dans la région d'Hamilton, notamment sur les services offerts à ces personnes immigrantes francophones dans une province très anglophone. À leur tour, elles démontrent comment la culture définit les codes de communication ainsi que les tabous, comme parler de viol de guerre, de ce que l'on a vécu de négatif. Dans la plupart des cultures, il n'est pas convenable de confier ses problèmes privés à une personne "étrangère ". Le plus souvent, les services sont organisés selon les concepts occidentaux et nord-américains, et non selon d'autres approches. Par exemple, la notion de santé mentale est une notion nord-américaine et peut paraître tout à fait inappropriée pour ces nouveaux immigrants qui ne demandent qu'à obtenir la résidence permanente avant de pouvoir envisager leur avenir. Avant de recourir à des services en santé mentale, ces personnes immigrantes recherchent une aide pour trouver du travail et pour faire face à leurs besoins de base, tels que trouver un logement et de la nourriture. Elles montrent également les limites des communautés culturelles et l'exclusion qui peut exister en leur sein, sur la base de facteurs divers, comme la religion, la langue, la couleur et l'appartenance à un groupe ethnique. Ce sont toutes ces dimensions que les intervenantes et intervenants doivent comprendre et intégrer à leurs interventions; ne pas les comprendre peut être très frustrant pour eux.

Peu d'organismes offrent des services spécialisés à ces populations traumatisées. C'est pour cela que les personnes immigrantes qu'ils servent doivent être invitées à s'investir dans ces organismes qui leur offrent des services, afin de mieux faire comprendre leur réalité et leur rationalité. Ce n'est qu'à ce moment-là que l'intervention pourra être mieux adaptée aux diverses réalités de ces populations traumatisées. 


\section{Des pratiques à notre image}

Quand ce sont les pratiques qui ne sont pas encore adaptées à ces nouvelles réalités issues de l'immigration et surtout des populations réfugiées, les enfants font face à de la discrimination dès l'école primaire. Se basant sur sa recherche dans les écoles primaires, Véronique Cloutier nous parle de l'intolérance ethnoculturelle que l'on y retrouve et de la perception, ou de l'absence de perception du problème, de la part des intervenantes ou intervenants. Pour ce faire, elle examine diverses facettes du racisme, n'hésitant pas à mentionner celui existant également entre les ethnies, et pas seulement celui des Canadiens vis-à-vis des enfants nés ailleurs. La couleur, la religion, la classe sociale et l'appartenance ethnique sont des facteurs utilisés par les enfants pour s'exclure mutuellement. Fait inquiétant, tous les enseignants et enseignantes ne voient pas le racisme exprimé par les enfants; certains le nient, parlant de farces. Cela indiquerait-il leur incapacité à identifier ce problème et, par conséquent, leur inaction pour l'enrayer? Ces enfants, ne disent-ils pas tout haut ce qu'ils pensent tout bas? Ces enfants ne reproduisent-ils pas le racisme prévalant dans leur milieu de vie? Car le racisme n'est pas inné, mais bien acquis. Parler de société multiculturelle signifie avant tout reconnaitre et accepter que le racisme existe et une volonté de l'éliminer, au même titre que toute autre discrimination. S'il faut combattre toute forme de discrimination dans la famille, il faut aussi que l'école devienne le lieu privilégié où les cultures se rencontrent et s'acceptent mutuellement.

\section{Collaboration spéciale}

Le même principe devrait s'appliquer à toute forme de discrimination, dont l'homophobie. C'est une autre forme de racisme et de violence par rapport à laquelle l'Europe vient de légiférer afin de la prévenir tant dans les relations interpersonnelles 
qu'institutionnelles et où beaucoup reste à faire, les hommes homosexuels étant encore largement victimes de discrimination. Il n'est pas loin le temps où un homosexuel, ou bien une personne identifiée comme telle, se faisaient congédier en toute impunité sur la base de son orientation sexuelle. Et il existe encore de nombreuses situations où ces homosexuels sont battus et même tués à cause de leur orientation sexuelle. C'est pour toutes ces raisons que nous avons sollicité Douglas Victor Janoff pour reproduire, avec l'accord des éditions Triptyque, le chapitre 4 de la version française de son livre Pink Blood - Homophobic violence in Canada, qui sortira cet automne sous le titre de Pink Blood - la violence homophobe au Canada. Son texte - Loi, homophobie et violence : légiférer pour punir les crimes motivés par la haine - démontre la violence systémique qui permet toujours aux agresseurs d'homosexuels d'agir et d'être relaxés en toute impunité. Se basant à la fois sur les articles parus dans les journaux, mais surtout sur les dossiers et jugements de tribunaux accessibles au public, il relève que bien souvent un double standard semble s'appliquer dans les cas d'agressions d'homosexuels. La police et les tribunaux ne les voient généralement pas comme des expressions de haine homophobe et ne donnent que des peines légères ou relâchent simplement les agresseurs qui feront alors de nouvelles victimes. Cette violence systémique fait que des coups, des blessures, des fractures et des meurtres passent presque sous silence, les agresseurs bénéficiant le plus souvent de circonstances atténuantes particulières, alors que les mêmes actes posés contre des personnes hétérosexuelles seraient jugés avec bien plus de sévérité. Des dossiers sont fermés, ajournés. Des personnes sont blessées et meurent parfois des conséquences de ces blessures, sans que justice leur ait été rendue ou ne le soit jamais, car il n'y a pas encore reconnaissance des crimes haineux contre les homosexuels et, par conséquent, il n'existe que peu de connaissances sur le sujet.

Quant à la violence faite aux femmes, même si c'est un sujet "classique ", il n'en demeure pas moins qu'elle n'a toujours pas disparu. Les services existants ne sont pas suffisants pour faire face à toutes les situations de violence touchant les femmes. Hors Québec, le problème se pose de façon aiguë aux Francophones; 
déjà insuffisantes, par rapport aux besoins criants en matière de protection, les ressources pour les femmes violentées francophones le sont encore plus. Ce point est bien démontré par Ghislaine Sirois et Marie-Luce Garceau quand elles parlent du développement des services en français en matière de violence faite aux femmes, à travers Action ontarienne contre la violence faite aux femmes (AOcVF). Mis sur pied en 1988, cet organisme provincial regroupe les maisons d'hébergement pour femmes victimes de violence, les centres d'aide et de lutte contre les agressions à caractère sexuel (Calacs) et quelques autres organismes offrant des SEF en matière de violence faite aux femmes.

S'il y a des acquis quant aux droits de la personne, il reste encore beaucoup à faire dans la société sur le plan des violences interpersonnelles et systémiques ainsi que sur le plan juridique et judiciaire pour faire disparaitre les multiples formes de violence encore socialement acceptées. 